\title{
Esclerosis múltiple, calidad de vida $y$ atención sociosanitaria
}

\author{
José Carrón \\ Sociólogo. Investigador social y consultor \\ pepecarron@gmail.com \\ Javier Arza \\ Departamento de Trabajo Social. Universidad Pública de Navarra \\ javier.arza@unavarra.es
}

Resumen: La esclerosis múltiple es una enfermedad neurodegenerativa. Se caracteriza por presentar una gran variabilidad de sintomas y por evolucionar en forma de crisis o brotes. Históricamente se ban observado divergencias en los datos sobre su prevalencia. Según los datos manejados en este estudio, puede afirmarse que la prevalencia se da, al menos, en 78 casos por cada 100.000 babitantes para Navarra, y que alcanza los 144 casos en el conjunto del Estado español. Esas cifras revelan que los datos utilizados tradicionalmente estaban notablemente por debajo de las cifras reales. En la actualidad no existe ninguna respuesta curativa para la esclerosis múltiple, pero sí opciones terapéuticas paliativas que alivian su cronicidad. En ese sentido, y como en todas las enfermedades crónicas, la mejora de la calidad de vida es hoy en día una cuestión de máxima importancia. La investigación nos muestra mejoras significativas principalmente en aspectos médico-sanitarios, pero también revela debilidades en otras áreas relacionadas con la autonomía y la movilidad de las personas afectadas, con su inserción en el ámbito laboral, con sus relaciones sociales, con la necesidad de mejorar el apoyo a su entorno familiar o con la imagen social. En la esclerosis múltiple emergen de manera simultánea necesidades sociales y sanitarias. En ese sentido, todo avance en el desarrollo de la atención sociosanitaria será positivo para las personas afectadas y para su entorno.

Palabras clave: Esclerosis múltiple, discapacidad, calidad de vida, sociosanitario. 
Multiple Sclerosis, Quality of Life and Socio-sanitary Care

Abstract: Multiple sclerosis is a neurodegenerative disease. It is characterized by a wide range of symptoms and evolves through episodic attacks. The data on its prevalence have varied historically. The data used in this study indicate that its prevalence in Navarre is at least 78 cases per 100,000 and that this climbs to 144 cases per 100,000 for Spain as a whole. These findings reveal that the real figures are significantly higher than the previous figures based on older data. There is currently no cure for multiple sclerosis but there are palliative treatment options. In this respect, as with all chronic diseases, improving quality of life has become a matter of the utmost importance. Research shows significant improvements mainly in medical and health care, but also reveals weaknesses in other areas affecting people with multiple sclerosis, such as autonomy and mobility, employment prospects, social relations, family support and social image. Multiple sclerosis generates new health and social needs simultaneously and so any advances in the development of medical and social care will benefit those affected and their environment.

Keywords: multiple sclerosis, disability, quality of life, social-healthcare. 


\section{Introducción}

A partir del impulso de la Asociación de Esclerosis Múltiple de Navarra (ADEMNA), y gracias a la financiación de la Fundación ONCE, durante el año 2011 desarrollamos un estudio cuyo objeto era el conocimiento de la situación y las necesidades de las personas afectadas por esta enfermedad en Navarra, además de la propuesta de acciones enfocadas a la mejora de su calidad de vida. En este trabajo presentamos los principales resultados de ese estudio, que ha combinado técnicas cuantitativas y cualitativas. Tras una aproximación a los conceptos relacionados con esta enfermedad y a una serie de datos epidemiológicos, presentaremos la metodología del estudio. Posteriormente abordaremos sus principales resultados, tanto los obtenidos a través del análisis cuantitativo (encuesta a personas afectadas) como los procedentes del análisis cualitativo (entrevistas y grupos focales con profesionales, familiares y miembros de ADEMNA), para abordar finalmente las conclusiones.

\section{Aproximación a la esclerosis múltiple}

\subsection{La esclerosis múltipleः concepto e impacto}

La esclerosis múltiple puede describirse, desde una perspectiva divulgativa, como un trastorno crónico del sistema nervioso central (formado por el encéfalo y la médula espinal) caracterizado por la pérdida progresiva de mielina. Esta es una sustancia blanca que actúa como una vaina recubriendo los nervios. Desde el momento en que va desapareciendo comienzan a producirse placas de tejido endurecido, proceso conocido como esclerosis. En consecuencia, los impulsos nerviosos se interrumpen periódicamente, y en ocasiones llegan a hacerlo de forma definitiva (Compston y Coles, 2008; Correale et alii, 2002).

Suele aparecer entre los 20 y los 40 años (Simone et alii, 2000), con una preponderancia en mujeres - en torno a dos terceras partes de los casos- (Duquette et alii, 1992) y es una enfermedad con una gran variabilidad en cuanto a los síntomas (EDE, 2003; ADEMNA, 2006; FELEM, 2007; FEDEMA, 2008), dependiendo de las áreas del sistema nervioso central que estén afectadas. Algunas manifestaciones clínicas frecuentes, que se presentan en forma de crisis o brotes, incluyen, según las ocasiones y los pacientes, parálisis, ataxia, temblores, debilidad, hormigueo, problemas visuales, pérdida de fuerza o sensibilidad, problemas en la función sexual, problemas de memoria a corto plazo, alteraciones urológicas, etc. (Raus, 1998; Delgado-Mendilívar et alii, 2005). 
La esclerosis múltiple es, habitualmente, una enfermedad con un largo recorrido. Su aparición en las primeras etapas de la edad adulta y su carácter no letal hacen que las personas que la padecen tengan por delante una larga esperanza de vida, durante la cual tendrán que convivir con la enfermedad y precisarán apoyo y cuidados por parte de su entorno, del sistema sociosanitario y de la sociedad en general.

A día de hoy no se dispone de ninguna respuesta curativa para la esclerosis múltiple, pero es cierto que se ha avanzado de manera notable tanto en el tratamiento como en la paliación de los síntomas, y se ha logrado detener la evolución de la enfermedad con intervenciones precoces mediante los tratamientos farmacológicos disponibles, aunque las secuelas no son reversibles (Banwell et alii, 2013; Giovannoni, 2013).

De todo ello no es difícil inferir, por un lado, el gran impacto emocional que tiene el diagnóstico y, por otro, su significativa repercusión en el proyecto vital de las personas afectadas y de sus familias: coincide con la etapa más productiva en la vida de una persona, supone una fuerte inversión de recursos (económicos, de tiempo, de esfuerzos...) y, en el caso de las mujeres, sobrerrepresentadas en esta enfermedad, implica una discriminación añadida a otras relacionadas con el género.

\subsection{La esclerosis múltipleः epidemiología}

Históricamente se ha dispuesto en España de numerosos estudios locales que, con diferentes metodologías, han ofrecido resultados significativamente divergentes entre sí sobre la prevalencia de la esclerosis múltiple (Mallada, 1999). A nivel estatal, la información epidemiológica que encontramos en diversas publicaciones del sector se mueve, generalmente, en estimaciones globales que se repiten de una a otra, sin establecer claramente su origen. Así, por ejemplo, en la introducción de una encuesta estatal realizada en 2005-2006 (FELEM, 2007) se dice textualmente: «en España, la prevalencia media se puede cifrar en 40-50 casos por cada 100.000 habitantes», aunque sin especificar el posible origen del dato. Otro tanto ocurre, por ejemplo, con la información, en general muy rigurosa, facilitada por el Instituto de Investigación de Enfermedades Raras en su página web: «en países mediterráneos la prevalencia es de 10-30/100.000 y en España se estima en cerca de 60/100.000, calculándose que hay unas 30.000 personas con esta enfermedad» (IIER, 2011). Sin embargo, si se acude directamente a fuentes estadísticas, en algún caso de origen secundario, como los registros del Departamento de Salud del Gobierno Foral de Navarra (2010), y en otros de origen primario, como la Encuesta del INE sobre discapacidad del año 2008 
(INE, 2013), los datos disponibles permiten establecer de manera más fidedigna unos niveles de impacto sensiblemente más elevados que los manejados hasta el momento.

Para Navarra, ámbito específico de la presente investigación, se dispone de información con metodología censal provista por el Gobierno Foral de Navarra (2010) sobre los casos que figuran en sus registros. Para 2010 son un total de 497 las personas diagnosticadas de esclerosis múltiple. Si se toma en cuenta la población de derecho para ese año en Navarra (636.924 habitantes), se obtiene una tasa de 78 casos por 100.000 habitantes, que situaría esta prevalencia en una zona de riesgo alto según la clasificación internacional establecida por Kurtzke $(1993,1997)$. En cuanto a la distribución por sexo, la sobrerrepresentación femenina que citábamos también se refleja en los datos navarros, donde la prevalencia entre ellas es de 121 casos por cada 100.000 mujeres, mientras que entre los varones es de 40 de cada 100.000 navarros.

Tabla 1. Personas afectadas por esclerosis múltiple en 2010 en Navarra.

\begin{tabular}{|c|c|c|c|}
\hline & Varones & Mujeres & Ambos sexos \\
\hline 0 a 14 & 0 & 1 & 1 \\
\hline 15 a 44 & 48 & 157 & 205 \\
\hline 45 a 64 & 60 & 171 & 231 \\
\hline 64 y + & 15 & 45 & 60 \\
\hline Total & 123 & 374 & 497 \\
\hline Casos $/ 100.000$ & 40 & 121 & 78 \\
\hline
\end{tabular}

Elaboración propia a partir de Gobierno Foral de Navarra (2010)

La distribución por edades responde, asimismo, a los patrones más conocidos para esta enfermedad (EDE, 2003; ADEMNA, 2006; FELEM, 2007; FEDEMA, 2008). La mayor parte (un 46,5\%) de los casos de 2010 en Navarra se sitúa en la franja de entre los 45 y los 64 años, a la que sigue la franja de 15 a 44 años (con el 41,2\%). Un 12,1\% tiene 64 años o más y solamente uno de los casos no había cumplido aún los 15 años.

Para la comparación de los datos navarros con el ámbito estatal nos hemos encontrado con serias dificultades ante la ausencia de datos globales provenientes de registros primarios. La aproximación más fiable de la que se dispone es la Encuesta EDAD 2008 (INE, 2013), en la que se entrevistó a más de 270.000 personas de 6 años o más. Aun siendo un volumen relativamente bajo de población $y$, por tanto, contar con un posible error de muestreo mayor, la estimación que 
realiza para el ámbito navarro es muy similar a los datos que se habían obtenido de fuentes primarias. Cifra los afectados por esclerosis múltiple en 78 casos por 100.000 habitantes, lo que representaría un total de 453 personas afectadas. Haciendo extensiva esa fiabilidad a los datos estatales (con mayor fuerza estadística al disminuir el error muestral por el tamaño considerablemente más elevado) se encuentran para la esclerosis múltiple 144 casos por 100.000 habitantes. La proyección en el conjunto de la población elevaría la cifra a más de 60.000 personas de 6 años o más afectadas por la enfermedad a nivel estatal, con una estimación estadística que, si acaso, podría estar sesgada a la baja en tanto que las personas afectadas con discapacidad leve o nula podrían no estar incluidas en estos resultados.

Tabla 2. Estimación de afectados por esclerosis múltiple en 2008 (6 años o más).

\begin{tabular}{|c|c|c|c|}
\hline & Varones & Mujeres & Ambos sexos \\
\hline España. Casos/100.000 & 83 & 204 & 144 \\
\hline Navarra. Casos/100.000 & 68 & 87 & 78 \\
\hline España. Total afectados & 17.300 & 43.600 & 60.900 \\
\hline Navarra. Total afectados & 199 & 254 & 453 \\
\hline
\end{tabular}

Elaboración propia a partir de encuesta EDAD (INE, 2008)

En los datos estatales de EDAD 2008 puede constatarse una vez más la contundente sobrerrepresentación de mujeres afectadas, en una relación de siete de cada diez casos (204 frente a 83 por 100.000 entre los varones). Para el caso específico de la población navarra, al segmentar por sexos, el notable aumento del error muestral en una población estadísticamente insuficiente impide valorar la falta de ajuste con los datos primarios ya comentados.

\subsection{Cambio de paradigma en el ámbito de la discapacidad}

$\mathrm{El}$ análisis de las consecuencias de patologías con un alto potencial discapacitante, como es el caso de la esclerosis múltiple, no puede llevarse a cabo sin hacer referencia al cambio de paradigma que está teniendo lugar en las últimas décadas en el ámbito de la discapacidad en su conjunto, y que se articula en torno a tres constructos altamente significativos:

+ El concepto de salud, en el que está en marcha un cambio de un modelo sanitarista a un modelo biopsicosocial que contempla aspectos como la prevención, el apoyo emocional, la salud pública o las modificaciones 
ambientales (OMS, 1978; Pineault, y Potvin, 2003; Ponte, 2008; OMS, 2009).

- Los servicios sociales y, en general, los derechos sociales, a través de un avance progresivo en la consideración de la atención a las necesidades sociales de la ciudadanía como un derecho subjetivo y de los servicios sociales como un sistema universal, garantista y de calidad (G. España, 2006; G. Navarra, 2006).

- La concepción y el abordaje de la discapacidad, que asiste a un cambio de enfoque desde un modelo individualista/asistencial/rehabilitador a un modelo inclusivo que busca, además de fomentar el trabajo individual, incidir sobre las creencias sociales y eliminar las barreras que impiden la participación social plena a las personas con discapacidad (Pérez, 2006; G. España, 2011; Huete y Díaz, 2009).

Es necesario poner de manifiesto que, en todo caso, el proceso de transformación iniciado a través de este cambio de paradigma no ha sido en el pasado lineal, sino que se ha enfrentado a numerosas dificultades, resistencias, contradicciones, etc. En estos momentos, la puesta en cuestión de aspectos nucleares del modelo sociosanitario permite incluso hablar de retrocesos significativos en su desarrollo.

En el ámbito de la esclerosis múltiple, la plasmación de este cambio de paradigma se ha reflejado de manera contundente en los Principios Internacionales para promover la calidad de vida de las personas con esclerosis múltiple (MSIF, 2002). Tomando como referencia la definición del concepto de calidad de vida por parte de la OMS como «percepción que tiene un individuo de su situación en la vida, dentro del contexto cultural y de valores en que vive y en relación con sus objetivos, expectativas, valores e intereses» (WHO, 1995), los parámetros médicos y asistenciales pasan a constituir un elemento más dentro de un planteamiento estratégico dirigido a lograr una mejora significativa en la calidad de vida de los afectados. Se estructura en torno a 10 principios, cuya sola enumeración nos pone en la pista de este cambio de enfoque: independencia y empoderamiento, asistencia médica, asistencia continua (cuidados de larga duración), promoción de la salud y prevención de la enfermedad, apoyo a miembros de la familia, transporte, empleo y actividades de voluntariado, pensiones y ayudas económicas por discapacidad, educación, vivienda y accesibilidad a edificios públicos.

Si bien la esclerosis múltiple supera el límite de 5 casos por cada 10.000 habitantes establecido en el marco de la UE para etiquetar una patología dentro del concepto de «enfermedades raras» (CCE, 2008), comparte con estas no pocas características y dificultades para su diagnóstico y abordaje. En el estudio citado promovido por la Federación Española de Enfermedades Raras (Huete y Díaz, 
2009) se hacía alusión a una imagen sencilla pero elocuente: «los afectados por estas enfermedades son peregrinos, viajeros permanentes en busca de un diagnóstico y tratamiento. Estas familias sufren de forma crónica las grandes dificultades de su enfermedad, agravadas por la escasez de dispositivos de ayuda».

\section{Material y método}

La metodología del estudio global ha sido mixta, combinando técnicas cuantitativas y cualitativas. El punto de partida se ha fundamentado en un amplio análisis documental de fuentes secundarias y una profunda investigación sobre la percepción y la dinámica social del concepto de discapacidad en su conjunto y sobre su plasmación concreta en el entorno de la esclerosis múltiple. A continuación se llevó a cabo una encuesta (cuya metodología se detalla posteriormente) a personas diagnosticadas en la Comunidad Foral de Navarra, con un total de 140 respuestas válidas. El cuestionario estaba centrado en revisar los principales datos sociodemográficos y médico-sanitarios de las personas entrevistadas, así como trazar un perfil de su situación en relación con los ítems incluidos en los Principios de Calidad de Vida relacionados con la esclerosis múltiple (MSIF, 2002). En la siguiente fase se realizaron 20 entrevistas semiestructuradas (15 a personas afectadas y 5 a profesionales sanitarios) y 6 grupos focales (profesionales del sistema sociosanitario, profesionales de ADEMNA, parejas de personas afectadas y padres o madres de personas afectadas). El estudio se finalizó con un análisis DAFO y la elaboración de una serie de recomendaciones y propuestas de avance.

\subsection{Resultados de la encuesta}

La encuesta propiamente dicha estaba dirigida a personas afectadas por la esclerosis múltiple en el ámbito de la Comunidad Foral de Navarra. Por cuestiones de confidencialidad respecto a cuestiones privadas de salud y, por ende, de posibilidad de localización, el universo poblacional se ha circunscrito a las personas socias de ADEMNA diagnosticadas con la enfermedad. Esto ha supuesto un total de 207 casos a los que nos hemos dirigido con una estrategia censal, con lo que no ha sido necesario hacer un diseño muestral representativo. El primer contacto fue realizado por ADEMNA que preguntó a los afectados si estaban dispuestos a participar en el estudio y, en caso afirmativo, si les parecía adecuada una entrevista personal o si preferían que les fuera enviado el cuestionario por correo para cumplimentarlo de manera privada.

El resultado final ha sido de 140 cuestionarios completados (un 67,6\% del universo fijado). Los casos no conseguidos lo han sido por las siguientes razo- 
nes: en un $16,9 \%$ el cuestionario postal no fue devuelto; en un $9,7 \%$ no se pudo localizar a la persona afectada; un 2,9\% rechazó participar en el estudio, y otro $2,9 \%$ estaba en un estado de salud que no permitía su participación. El $65 \%$ de los cuestionarios se cumplimentó mediante entrevista personal y el $35 \%$ restante fue cumplimentado por las personas encuestadas y devuelto por correo.

El trabajo de campo fue realizado entre el 5 de septiembre y el 30 de noviembre de 2011 por un equipo de cinco profesionales supervisados por el equipo investigador. La explotación de los datos se ha llevado a cabo con el paquete estadístico SPSS 17.0.

\subsection{Perfil sociodemográfico}

El perfil sociodemográfico que se refleja a partir de la encuesta ofrece los siguientes resultados: la edad media de las personas afectadas por esclerosis múltiple es de 46,5 años, con un rango que oscila entre los 25 y los 80 años, pero en el que seis de cada diez afectados están ubicados en la horquilla de 35 a 54 años. Por sexos, siete de cada diez personas afectadas (70,7\%) son mujeres.

Viven básicamente en pareja y solamente un $8 \%$ lo hacen solos, frente al porcentaje del 18,8\% en la población general española (INE, 2011). El 64,3\% ha tenido hijos, y destaca el hecho de que cuatro de cada diez (un 38,9\%) los han tenido cuando la enfermedad ya había sido diagnosticada.

Solamente un 4,3\% no había conseguido terminar los estudios primarios, un $32,1 \%$ sale del sistema educativo con estudios primarios, un 32,1\% con educación secundaria finalizada y el $31,4 \%$ ha obtenido alguna titulación universitaria.

En cuanto a su situación laboral, tres de cada diez (un 30,7\%) trabajaban fuera del hogar, uno de cada diez (el 11,4\%) se encontraba en desempleo, la tercera parte (el 33,6\%) recibía una pensión de invalidez/incapacidad permanente, el 12,8\% recibía una pensión de jubilación y un 7,9\% se dedicaba a las tareas del hogar. La media de ingresos mensuales por unidad familiar es de 2.040 euros, pero la distribución es notablemente heterogénea: tres de cada diez hogares no superan los 1.200 euros de ingresos; aproximadamente la cuarta parte (el 23,6\%) tiene unos ingresos entre 1.200 y 1.800 euros y un porcentaje similar (el 26,4\%) entre 1.800 y 3.000 euros. Para el conjunto de la población navarra, la renta media por hogar en 2010 era de 2.867 euros (INE, 2010). De hecho un 52,1\% declaraba no tener dificultades para «llegar a fin de mes», mientras que en el conjunto de los hogares navarros ese porcentaje se elevaba al 65,5\% (INE, 2010). 


\subsection{Perfil médico-sanitario}

A ocho de cada diez encuestados (un 80,8\%) se les diagnosticó esclerosis múltiple entre los 20 y los 44 años, y solamente en un 4,3\% de los casos se detectó la enfermedad antes de dicha edad. El diagnóstico en los varones es más precoz, como puede apreciarse en el gráfico adjunto.

Ilustración 1. Diagnóstico en función de la edad y sexo.

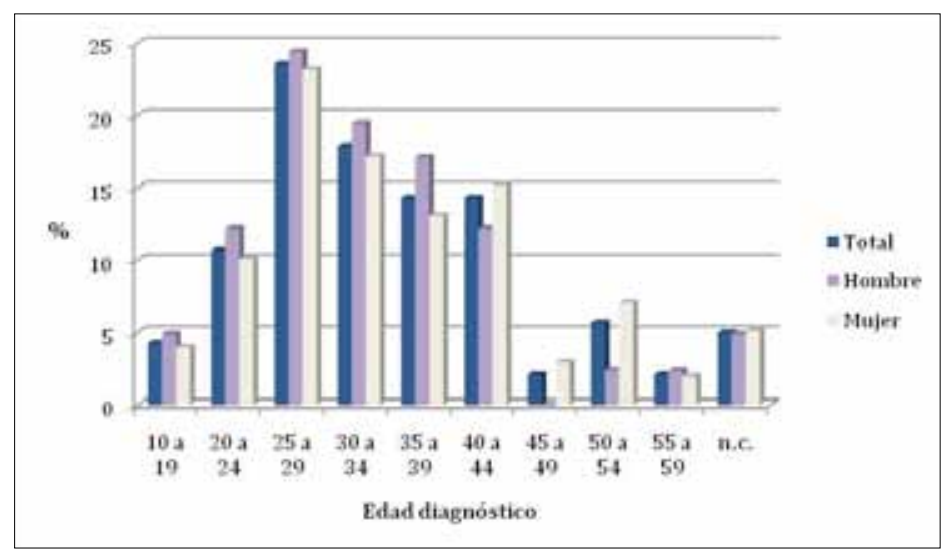

Elaboración propia

La mitad de las personas encuestadas (un 49,9\%) llevaba entre 5 y 15 años diagnosticada; un 13,6\%, entre 16 y 20 años, y en un 17,1\% de los casos habían pasado más de 20 años desde el diagnóstico. Seis de cada diez personas afectadas (un 61,4\%) tenían reconocido algún grado de minusvalía: en el 39,5\% de los casos esta se situaba entre el $33 \%$ y el $64 \%$, en el $37,2 \%$ de los casos era del $65 \%$ o superior y en un $23,3 \%$ era menor del $33 \%$. La diferencia por sexos es significativa: el $41,7 \%$ de las minusvalías femeninas era del $65 \%$ o superior, mientras que en el caso de los varones el porcentaje era del $26,9 \%$.

Los síntomas asociados a la esclerosis múltiple más citados por los encuestados (los padecen siempre o a menudo) son la fatiga, en el $72 \%$ de los casos; la falta de equilibrio, en un $62,1 \%$, y los síntomas motores, en un 56,5\%. Igualmente, casi la mitad manifiestan dificultades sensitivas o urinarias y espasticidad muscular. Con porcentajes menores, pero también altamente significativos, se señalaron disfunciones sexuales, síntomas visuales, dolor, dificultad de control de esfínteres y otros síntomas. Es especialmente significativa la diferencia por sexos en los síntomas de dolor: la población masculina manifiesta sufrirlo siempre o a menudo en un $36,6 \%$ de los casos, mientras que en las mujeres el porcentaje es del 20,3\%. 
Un 13,6\% afirmaba tener un familiar consanguíneo que también padecía esclerosis múltiple, siendo una especificidad notablemente más presente en la población femenina (un 16,2\%) que entre los varones (ocurría en el 7,3\% de los casos).

Casi la quinta parte (un 19,3\%) manifestaba no recibir ningún tratamiento para la enfermedad, situación sensiblemente más frecuente entre los varones (un $26,8 \%$ no seguía ningún tratamiento farmacológico) que entre las mujeres (así ocurría en un $16,2 \%$ de la población femenina).

\section{Ilustración 2. Evolución del porcentaje de afectados con tratamiento farmacológico.}

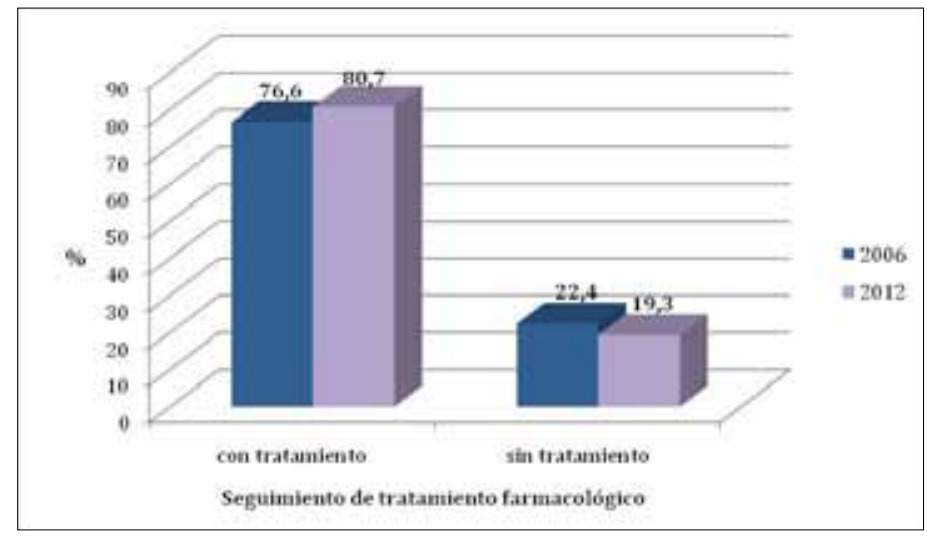

Elaboración propia

En el caso de que la respuesta fuera positiva respecto a los tratamientos farmacológicos, los tratamiento más utilizados para la esclerosis múltiple eran Tysabri (un 18,6\%), Copaxone (un 17,1\%), Avonex y Rebif (ambos en un 12,9\% de los casos). Por otro lado, dos tercios de las personas encuestadas siguen algún tratamiento farmacológico en relación con los síntomas asociados. Los más frecuentes están relacionados con la espasticidad (un 30\% de los afectados la maneja con algún tipo de fármaco), con la fatiga (un 26,4\% lo hace), con el dolor (un $22,1 \%$ ), con la depresión (un 22,1\%) o con el insomnio (un 21,4\%). La diferencia más significativa por sexos se producía en relación con la depresión: un 25,3\% de la población femenina seguía un tratamiento mientras que solamente lo hacía un $14,6 \%$ de la masculina. Al igual que en los tratamientos específicos para manejar la enfermedad, en relación con los síntomas asociados es superior el porcentaje de varones que no siguen un tratamiento (el 43,9\%) que el encontrado entre las mujeres (un 31,3\%). 
Los tratamientos alternativos son utilizados por más de un tercio de las personas afectadas (el 35\%), siendo los más frecuentes la herboristería (un 15\% afirmaba utilizarla), los cuidados nutricionales (un 14,3\%), la homeopatía (un 9,3\%) y la acupuntura (el 7,1\% de los casos).

En el 53,6\% de los casos la persona entrevistada no sabía si le habían aplicado la escala EDSS (Kurtzke, 1983), considerada una medida de referencia; un 15\% confirmaban que sí la habían pasado aunque solamente el 71,4\% de estos conocía los resultados. Más de la mitad de los casos (un 53,3\%) habían obtenido valores que oscilaban entre $4 \%$ y $5 \%$.

\subsection{3 Áreas de calidad de vida}

Independencia y empoderamiento. El 43,6\% necesita ayuda de otras personas para realizar alguna de las actividades básicas de la vida diaria, situación más frecuente entre la población femenina (un 49,5\%) que entre la masculina (el 29,3\%).

\section{Ilustración 3. Necesidad de ayuda para las actividades de la vida diaria.}

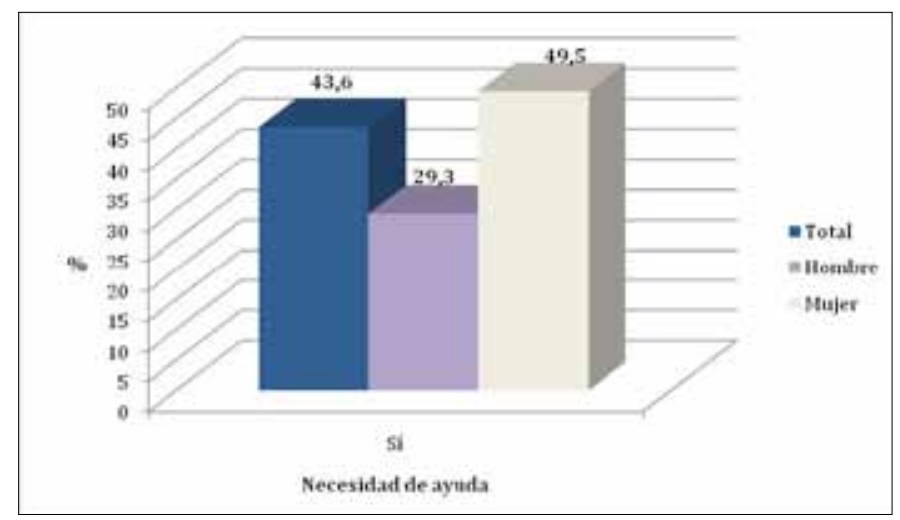

Elaboración propia

Entre quienes necesitan ayuda, las necesidades vitales más frecuentes están relacionadas con los desplazamientos fuera del hogar (un 52,5\%), con bañarse (un 39,4\%), con vestirse (un 29,5\%), o con levantarse/acostarse (un 27,8\%). Por lo que se refiere a otro tipo de necesidades no vitales pero importantes, destacan la ayuda para poder ir de compras (el 63,9\%necesitaba ayuda), para la limpieza de la casa (un 59\%), para la realización de gestiones y papeleo (un 47,5\%) o para preparar la comida (un 32,8\%). Un 44,3\% afirma utilizar alguna ayuda técnica. Tres de cada diez (un 30\%) utilizan muleta/bastón; un 17,9\%, una silla manual; el 9,3\%, una silla a motor; un $5,7 \%$, un andador, y un 6,4\%, algún otro tipo de 
ayuda técnica. Casi las dos terceras partes de quienes las utilizan (un 64,5\%) manifiestan hacerlo de forma permanente. Un 30\% declara tener tarjeta de estacionamiento para minusválidos y otro $12,1 \%$ dice necesitarla aunque no dispone de ella.

Asistencia continua. La gran mayoría (un 85\%) declara que necesita algún tipo de servicio de apoyo. Este tipo de servicio es significativamente más reclamado por la población femenina (un $89,9 \%$ frente al $73,2 \%$ entre los varones). Los servicios más demandados son fisioterapia (por un $58,6 \%$ de los encuestados), yoga/pilates/estiramientos (por el 45\%), más información sobre la enfermedad (por un 28,6\%), servicio de ayuda a domicilio (un 23,6\%) o servicio de viajes y vacaciones con apoyo (un $22,1 \%$ ).

Promoción de salud y calidad de vida. Un 42,1\% considera que su salud durante los últimos doce meses ha sido buena mientras que un $17,1 \%$ la considera mala. Entre quienes llevan menos años con la enfermedad diagnosticada es más bajo el porcentaje que valora positivamente su salud (un 33,3\% para menos de 5 años, un $44,8 \%$ entre 5 y 15 años, y un $41,9 \%$ para más de 15 años). Por otro lado, la valoración negativa es notablemente más intensa entre las mujeres (un 21,2\%) que entre la población masculina (solamente el 7,3\% la considera mala). En una escala de 1 (muy negativa) a 10 (muy positiva), la puntuación media respecto a la calidad de vida de la que disfrutan se sitúa en 6,5 puntos. Un 35\% ha recibido ayuda psicológica en alguna ocasión: un 16,4\% la utiliza de manera puntual, un $13,6 \%$ la recibió solamente cuando le diagnosticaron la enfermedad y el 5\% la recibe de manera continua desde el diagnóstico.

Transporte. Un 86,3\% tiene vehículo propio. De estas personas, un 61,5\% manifiesta que no necesita ninguna adaptación y un 28,2\% sí tiene realizadas las adaptaciones necesarias para su manejo. El resto, aunque las necesitaría, no dispone de ellas. Solamente un $8,5 \%$ de las personas propietarias de vehículos había solicitado alguna subvención para llevar a cabo tales adaptaciones.

Vivienda y accesibilidad. El 28,8\% dice que el acceso a su vivienda no ha necesitado ningún tipo de adaptación. Por el contrario, un $47,5 \%$ declara que ya ha sido adaptado, mientras el 23,8\% manifiesta que la accesibilidad de su hogar no está adaptada a las necesidades derivadas de su enfermedad. En lo relacionado con la adaptación de la vivienda en sí, un 42,4\% ya ha realizado las adaptaciones necesarias, mientras que otro $12,9 \%$ expresa que sería necesario realizar dichas adaptaciones. La falta de adaptación tanto de los accesos como de la propia vivienda está relacionada, en primer lugar, con problemas económicos y, en segundo lugar, con problemas técnicos de la vivienda. 
Empleo. El 30,7\% estaba trabajando activamente, un 46,4\% tenía reconocida una incapacidad laboral, un $11,4 \%$ se encontraba en situación de desempleo y el resto estaba en otras situaciones (ocupándose de las tareas del hogar, estudiando, etc.). La actividad laboral se reduce notablemente a medida que aumenta la edad (el 55\% en el tramo de 25 a 34 años y solamente el 7,7\% en el de 55 a 64 años). El $22,1 \%$ de las personas encuestadas tuvieron problemas en su trabajo al conocerse su diagnóstico, siendo los más frecuentes el despido (en un 41,9\% de los casos), la incomprensión y pérdida de confianza (en el 22,6\%) o la presión para dejar el trabajo (en un 12,9\%). Los ingresos de la unidad familiar proceden en un 18,6\% exclusivamente de la persona afectada, mientras que en un $62,9 \%$ de los casos los ingresos de la persona afectada son una parte más, que se suma a la contribución de otros miembros del hogar.

Pensiones y ayudas. Un 18,6\% de las personas entrevistadas tenía realizada una valoración de su dependencia de acuerdo con la normativa vigente, y otro 2,1\% estaba a la espera de obtenerla. Esta valoración era notablemente más frecuente a medida que avanzaba la edad: era de un $5 \%$ en el tramo de edad de 25 a 34 años y llegaba hasta el 30\% entre los encuestados de 65 años o más. De las personas a las que se había realizado esta valoración, un $57,7 \%$ pertenece al primer grado o «dependencia moderada» (38,5\% para el nivel I y 19,2\% para el nivel II); un 26,9\%, al segundo grado o «dependencia severa» $(19,2 \%$ y $7,7 \%$ respectivamente), y un 15,3\%, al tercer grado o "gran dependencia» $(11,5 \%$ y $3,8 \%$ respectivamente para los niveles I y II). Solamente un 13,6\% del total de personas entrevistadas afirma recibir algún servicio o prestación económica por su dependencia. Las principales prestaciones son ayudas económicas para asistencia personal (un 52,6\%), y servicio telefónico de emergencias (un 26,3\%).

\subsection{Resultados del análisis cualitativo}

El análisis cualitativo nos ha permitido aproximarnos al punto de vista de los agentes más significativos en el ámbito de la esclerosis múltiple:

+ Personas diagnosticadas.

+ Profesionales sanitarios y sociosanitarios.

- Profesionales que desempeñan labores de planificación y gestión en los ámbitos de la discapacidad, la incapacidad laboral y la dependencia.

- Familiares de personas diagnosticadas.

+ Miembros de la asociación.

Para el análisis cualitativo hemos utilizado dos tipos de técnicas: entrevista semiestructurada y grupo focal. La entrevista semiestructurada, que suele tener aproximadamente una hora de duración, tiene como cometido el acceso a la pers- 
pectiva del sujeto estudiado. El entrevistador/a dispone de un guión, que recoge los temas que debe tratar a lo largo de la entrevista, pero lo maneja con gran flexibilidad para facilitar la libertad de expresión. En cuanto al grupo focal, su duración aproximada es de hora y media. Consiste en reunir a un grupo de entre seis y ocho personas, guiado por un o una profesional que actúa como facilitador/a de las discusiones. $\mathrm{Al}$ igual que hemos señalado en el caso de las entrevistas, la persona facilitadora utiliza un guión para mantener el enfoque y el control de la reunión, pero debe manejarlo con mucha flexibilidad.

\subsubsection{Sintesis del análisis cualitativo}

Grupo focal con profesionales de planificación y gestión de la discapacidad y la dependencia

- El modelo de valoración, especialmente en la evaluación de la discapacidad y la incapacidad laboral, está muy orientado por «parámetros objetivos» que utilizan escalas para medir fundamentalmente la disfunción motriz permanente. Sin embargo, gran parte de la sintomatología más característica de la esclerosis múltiple no es permanente y es difícil de cuantificar por la ausencia de escalas de medición.

- En el discurso del ámbito de la dependencia y los servicios sociales se detecta una mayor sensibilización hacia la consideración de parámetros de carácter más «subjetivo». Se afirma, además, que ya hay experiencias al respecto con otro tipo de enfermedades.

- En el discurso del grupo aparecen también las dificultades del espacio sociosanitario. Si no es abordado adecuadamente (desde las metodologías, pero también desde las actitudes), el espacio puede ser más de desencuentro que de construcción. Asimismo, se alude a la necesidad de una adecuada financiación.

- El discurso es unánimemente crítico respecto a la Ley de la Dependencia: fomenta prestaciones de carácter asistencial, pero no aquellas que buscan promover la autonomía personal.

- Se destaca también la necesidad de impulsar medidas en el ámbito laboral.

\section{Entrevistas a profesionales sanitarios}

- Se coincide en que la atención sanitaria y farmacológica es buena. Se valora que la atención es más personalizada y con un mejor seguimiento de la enfermedad. 
- Las principales «asignaturas pendientes» sonः diagnóstico precoz, rehabilitación (física y cognitiva) y tiempo de dedicación a pacientes.

- Las principales dificultades de las personas afectadas son: aceptación de la enfermedad; pérdida del equilibrio, déficit de fuerza y deterioro cognitivo (en lo físico); depresión y tendencia a «encerrarse en sí mismo» (en lo psicológico), e integración social y laboral (en lo social).

- La labor informativa con el o la paciente es de gran relevancia. Debe iniciarse en el momento del diagnóstico, pero ser continuada en las revisiones.

- Se manifiesta la necesidad de una mayor concienciación social de la ciudadanía.

- Los pilares de la actuación son dos: la asistencia hospitalaria y la asistencia social (centrada fundamentalmente en ADEMNA). En este sentido, la asociación es una pieza clave en el proceso de atención, según el discurso recogido en las entrevistas.

Entrevistas a personas a las que se ha diagnosticado esclerosis múltiple

- Una atención de calidad debe reunir las siguientes características: accesibilidad, cercanía y confianza. En general, consideran que estos criterios son cumplidos por sus profesionales de referencia (tanto del sistema sanitario como de ADEMNA).

- Uno de los síntomas más mencionados es la fatiga. Asimismo, se hace alusión a los efectos secundarios de la medicación.

- La incertidumbre está muy presente en la vida de las personas afectadas.

- Las claves para una buena adaptación son: tener una buena información, aceptar las posibles limitaciones y adaptarse a ellas desde la vivencia del «día a día».

- Respecto a la información, se valora de una manera muy positiva la posibilidad de tener espacios de feedback con el personal sanitario. La web de ADEMNA y las conferencias que organiza se señalan como fuentes de confianza.

- Las experiencias de maternidad tras el diagnóstico se valoran positivamente, pero suponen sobrecarga (también para el entorno).

- El ámbito laboral se señala como un espacio de vulnerabilidad. Se subraya la necesidad de sensibilizar a las empresas para facilitar la contratación y la adaptación de puestos.

- El apoyo más valorado es el que procede del entorno familiar. 
- Se detecta una gran desconfianza hacia las valoraciones de discapacidad, incapacidad laboral y dependencia realizadas desde la Administración Pública.

- El apoyo de ADEMNA es complementario al recibido por la red natural de apoyo. Desde el discurso detectado, la clave reside en saber combinar adecuadamente ambos.

Grupos focales con familiares

- El proceso de aceptación y adaptación a la enfermedad debe ser realizado por la persona afectada, pero también por su familia.

- De una manera similar a como surgió en el discurso de las personas afectadas, para los familiares la adaptación es una combinación entre el reconocimiento de las necesidades y limitaciones, la valoración de aspectos positivos y negativos, la búsqueda de posibilidades de mejora y la orientación hacia el «día a día».

- En el proceso de aceptación-adaptación es fundamental delimitar espacios, que la vida no gire en torno a la esclerosis múltiple, que no se mimeticen los estados de ánimo. En el caso de progenitores, el apoyo de la pareja es fundamental para ello.

- Otra de las claves reside en contar con apoyos externos: formación, productos de apoyo, orientación, etc. En ese sentido, se demanda una mayor atención específica desde la asociación. No obstante, también se reconoce que es costoso admitir la necesidad de ayuda y pedirla.

- A pesar de todo, la sensación de incertidumbre y los sentimientos de impotencia, sobrecarga, etc. están más o menos presentes en la vida de los cuidadores y cuidadoras.

- La valoración de ADEMNA es muy alta. Como propuesta de mejora, se resalta especialmente la necesidad de una mayor visibilidad social, especialmente importante en el caso de una enfermedad tan desconocida como esta.

- En cuanto a las administraciones públicas, se alude a su falta de conocimiento sobre la realidad de las personas afectadas y a cómo ADEMNA cubre sus carencias.

Profesionales de ADEMNA

- La calidez, la cercanía y la disponibilidad son los valores que encarnan la atención de calidad para el equipo. La profesionalidad, la atención inte- 
gral, la innovación y la interdisciplinariedad se presentan como valores complementarios.

- En el equipo se detectaron dos visiones distintas: una que espera abordar la sensación de saturación a través de «recursos externos» y otra que confía más en la mejora de la eficiencia.

- Trabajar en ADEMNA significa enfrentarse a momentos de frustración e impotencia, por las características de la enfermedad. El equipo se valora como la herramienta fundamental para abordar estos costes emocionales.

+ El equipo percibe un clima de compromiso, implicación y creatividad.

- Se valora que la relación con el sistema sanitario ha mejorado. No obstante, es necesario seguir avanzando hacia una mejora en la coordinación de casos.

\section{Conclusiones}

La evolución en el paradigma desde el que se contempla la discapacidad en general y, en concreto, la esclerosis múltiple, ha estado marcada por los cambios en el concepto de salud, en el avance de los derechos sociales subjetivos y en la implantación progresiva de un modelo inclusivo para su abordaje. Todo ello, como hemos podido constatar en la investigación, ha llevado a cimentar un constructo en el que los aspectos sanitarios y asistenciales son una parte significativa, pero solo una parte de un abordaje integral. En todo caso, la puesta en cuestión en el momento actual en toda Europa, y en España en particular, de las políticas de bienestar deja en una situación de incertidumbre su dirección futura.

La mayor parte de las investigaciones realizadas en España se han dado a nivel local, con una horquilla muy dispersa en cuanto a la prevalencia de la enfermedad. A nivel estatal se ha comprobado que las cifras que circulaban hasta ahora estaban notablemente por debajo de las cifras reales. Partiendo tanto de los registros primarios de Navarra como de la encuesta EDAD 2008, y contrastando sus resultados, puede afirmarse que la prevalencia sería, al menos, de 78 casos por 100.000 habitantes para la Comunidad Foral de Navarra y que alcanzaría los 144 casos en el conjunto de España (entre la población de 6 años o más).

Desde un punto de vista metodológico se ha coincidido con las principales investigaciones con objetivos similares, en lo que se refiere la limitación del universo de la encuesta a las personas diagnosticadas que integran las asociaciones del ámbito de referencia. Aún así, para el caso de Navarra se ha dispuesto de un «N» de partida que alcanzaba casi la mitad de quienes figuraban en los registros 
oficiales y, por otra parte, el nivel de respuesta se sitúa en la franja alta de los obtenidos en estudios similares.

En la comparación con otros estudios, el perfil sociodemográfico de las personas con esclerosis múltiple permanece bastante estable: la media de edad es ligeramente inferior a los 50 años, en torno a dos tercios son mujeres, menos de una de cada diez viven solas, la tercera parte recibe una pensión de incapacidad y más de la mitad de los hogares no superan unos ingresos de 1.800 euros mensuales. Sin embargo, las diferencias sí son destacables respecto a la población general, entre los que, por ejemplo, se duplica el porcentaje de hogares individuales o disponen de una renta media de 2.867 euros mensuales por hogar. Este contraste permite visualizar el importante grado incapacitante de la enfermedad, con un grave impacto laboral (que también ha sido señalado en el análisis cualitativo) y, por ende, con su correlato socioeconómico.

En lo referido al perfil médico-sanitario, se confirma un diagnóstico mayoritario entre los 20 y 44 años y que seis de cada diez afectados tienen reconocido algún tipo de minusvalía (en más de un tercio de los casos igual o superior al $65 \%$ ). También se confirman la fatiga, la falta de equilibrio y los síntomas motores como los más frecuentes entre las personas afectadas. Sin embargo, aumentan de manera significativa las personas afectadas que siguen un tratamiento farmacológico: solamente dos de cada diez no lo hacen en la actualidad (situación más frecuente entre los varones). Por otra parte, el recurso a terapias «alternativas» sigue siendo utilizado por más de un tercio de las personas afectadas, especialmente la herboristería, los cuidados nutricionales, la homeopatía y la acupuntura.

Como podía deducirse de los síntomas y del curso de la esclerosis múltiple, más allá del estricto espacio clínico se encontró toda una serie de dificultades relacionadas con el entorno que inciden directamente en la calidad de vida del colectivo. Se destacan de manera especial algunas cuestiones:

- En relación con su autonomía, casi la mitad de las personas encuestadas necesitan ayuda de otras personas para realizar algunas de las actividades básicas de la vida diaria, especialmente desplazarse fuera del hogar, bañarse, vestirse y levantarse/acostarse. Las cifras relacionadas con este hecho permanecen estables respecto al estudio de 2006. Aumenta, incluso, la cifra de quienes utilizan alguna ayuda técnica (también casi la mitad de las personas encuestadas), destacando muletas/bastones y sillas manuales, y las dos terceras partes de los que utilizan estas ayudas técnicas lo hacen de manera permanente.

- Más allá de las tareas personales, ocho de cada diez necesitan utilizar servicios de apoyo, destacando especialmente los de fisioterapia, si bien 
la presencia de estos últimos desciende ligeramente respecto a 2006. En cambio, está en ascenso la utilización de servicios de yoga/pilates/estiramientos o de ayuda a domicilio.

- La percepción del propio estado de salud es un indicador importante de la vivencia personal de la enfermedad. Si bien cuatro de cada diez personas afectadas consideran que su salud ha sido buena en el último año, hay que destacar que esta frecuencia es directamente proporcional al número de años pasados desde el diagnóstico. El impacto del diagnóstico tiene un importante efecto negativo en esa percepción, que va atemperándose con el tiempo, posiblemente a raíz de un mayor conocimiento de la enfermedad y de la adquisición de habilidades personales para su manejo. De hecho, más de la tercera parte de afectados ha recibido ayuda psicológica en alguna ocasión.

- La mitad de las personas encuestadas había tenido que adaptar el acceso a su vivienda, pero una cuarta parte declara que el acceso a su vivienda no está adaptado, aun necesitándolo. También cuatro de cada diez tuvo que adaptar la propia vivienda y uno de cada diez necesitaría hacerlo y no lo ha hecho, básicamente por dificultades económicas. Llama poderosamente la atención que esta última necesidad se eleva a más de un tercio de las personas encuestadas en el estudio estatal llevado a cabo por FELEM en 2006, sin que haya evidencias para aventurar si la diferencia se debe a una evolución positiva o una cuestión de ámbito de la investigación.

- Las dificultades de movilidad y la falta de adaptación del transporte público dan lugar a que la inmensa mayoría disponga de vehículo propio. Si bien tres de cada diez lo han adaptado a sus necesidades, todavía uno de cada diez no lo ha hecho, aunque lo necesitaría y, al igual que en el caso de la vivienda, las razones son fundamentalmente económicas.

- En el ámbito laboral, tres de cada diez encuestados estaban en situación de ocupación y uno de cada diez se encontraba en situación de desempleo, mientras que casi la mitad de las personas encuestadas tenía reconocida una incapacidad laboral. Lamentablemente, un dato que se mantiene estable respecto al estudio de 2006 es que más de la quinta parte tuvieron problemas en su lugar de trabajo al conocerse el diagnóstico de su enfermedad.

- A pesar de las carencias del sistema de valoración de la dependencia para comprender la especificidad de la esclerosis múltiple, dos de cada diez personas encuestadas tienen realizada una valoración. De ellas, la cuar- 
ta parte se reconoce como casos de dependencia severa (grado 2), y un 15,3\%, como casos de gran dependencia (grado 3). La falta de adaptación a las necesidades de la enfermedad también se pone en evidencia en el tipo de ayudas prestadas: más de la mitad es una ayuda económica y una cuarta parte se presta en forma de teleasistencia.

En conclusión, estamos ante una enfermedad frente a la que hoy por hoy no hay una respuesta curativa, con opciones terapéuticas paliativas que alivian su cronicidad, y en la que los aspectos que inciden en la mejora de la calidad de vida son fundamentales. $Y$ en ese reto no basta con incidir en el ámbito individual, sino que las intervenciones en el entorno resultan esenciales para avanzar.

Los resultados de la investigación llevada a cabo nos muestran mejoras significativas en aspectos médico-sanitarios y en otras áreas, pero también déficits importantes, sobre todo en lo relacionado con la autonomía y la movilidad de las personas afectadas y con su ámbito laboral y relacional. Resulta necesario, asimismo, aumentar la «visibilidad» de la esclerosis múltiple, especialmente de cara a la valoración de la dependencia y las necesidades de los afectados, dificultada por el carácter intermitente de la enfermedad. En pocas palabras, es necesario posibilitar la integración activa y plena en la sociedad de las personas con esclerosis múltiple.

Especialmente relevantes resultan las necesidades de intervención en el ámbito familiar. Como hemos recogido en el análisis cualitativo, la esclerosis múltiple afecta al sistema familiar en su totalidad. Para que su impacto no provoque disfunciones, es imprescindible que se desarrollen políticas de apoyo a los familiares. Estas políticas deben tener en cuenta que el cuidado y apoyo prestado por familiares es una pieza estratégica que debe formar parte del continuo asistencial desarrollado desde el sistema sociosanitario. Por ello, deben existir apoyos (formativos, psicológicos, económicos, de respiro familiar, técnicos, a domicilio, etc.) que faciliten un cuidado familiar efectivo y que prevengan la sobrecarga de las personas cuidadoras y otros impactos negativos en el sistema familiar. Asimismo, se debe prever un mayor desarrollo de los recursos sociosanitarios que permita el cuidado integral y de larga duración a personas con discapacidad que no cuenten con el apoyo familiar necesario.

Sería interesante que futuros estudios sobre la situación y necesidades de las personas afectadas por esclerosis múltiple, si no resultase factible que tuvieran una representatividad estatal, se llevasen a cabo en sus respectivos ámbitos con metodologías que permitan su comparación. Una mejora significativa sería la inclusión, en el universo de estudio, de personas afectadas que se encuentren fuera de los ámbitos asociativos. 


\section{Bibliografía}

ADEMNA (2006). Esclerosis múltiple en Navarra: realidad, necesidades sociales $y$ calidad de vida. Pamplona: Asociación ADEMNA.

Banwell, B.; Giovannoni, G.; Hawkes, C.H.; Lublin, F. (2013) «Editor's welcome and a working definition for a multiple sclerosis cure». Mult Scler Relat Disord (Netherlands); 2 (2): 65-7.

Comisión de las Comunidades Europeas (2008). «Comunicación de la Comisión. Las enfermedades raras: un reto para Europa». Bruselas: COM (2008) 679 final.

Compston, A., Coles, A. (2008). «Multiple sclerosis». Lancet, 372 (9648): 1502-17.

Correale, J.; Arriaga, C.; López, L.; Meli, F.; Ochoa, S.; Tenembaum, S. (2002). «Enfermedades desmielinizantes». En: Micheli, F; Nogués, M.; Asconapé, J.; Fernández, M.; Biller, J. (dirs.). Tratado de neurología clínica. Buenos Aires. Editorial Médica Panamericana. p. 642-96.

Delgado-Mendilívar, J.M.; Cadenas-Díaz, J.C.; Fernández-Torrico, J.M.; Navarro-Mascarell, G.; Izquierdo, G. (2005). «Estudio de la calidad de vida en la esclerosis múltiple». Rev Neurol., 41: 257-62.

Duquette, P.; Pleines, J.; Girard, M.; Charest, L.; Senecal-Quevillon, M. y Masse, C. (1992). «The increased susceptibility of women to multiple sclerosis». Can J Neurol Sci., 19: 466-71.

EDE Fundazioa (2003). Las personas afectadas de esclerosis múltiple y sus necesidades: Estudio de los y las asociadas a la Fundación Esclerosis Múltiple. Eugenia Epalza Fundazioa. Bilbao: EDE Fundazioa.

European Multiple Sclerosis Platform (2008). Code of good practices in multiple sclerosis. Brussels: EMSP.

FEDEMA (2008). Atención social, estudio de necesidades y costo socio-laboral de la esclerosis múltiple y otras enfermedades similares. Sevilla: Federación FEDEMA.

FELEM (2007). Esclerosis múltiple en España: realidad, necesidades sociales y calidad de vida. Madrid: Real Patronato sobre Discapacidad.

Giovannoni, G. (2013). «Ectrims 2012: Treatment Highlights». Mult Scler Relat Disord., 2 (2): 68-72

Gobierno de España (2006). «Ley 39/2006, de 14 de diciembre, de Promoción de la Autonomía Personal y Atención a las personas en situación de dependencia». Boletin Oficial del Estado, 299 (15 dic 2006) Disponible en: <http://www.boe.es/boe/dias/2006/12/15/pdfs/A44142-44156.pdf> 
Gobierno de España (2011). Ministerio de Sanidad, Política Social e Igualdad. «Estrategia Española sobre Discapacidad 2012-2020». Madrid: Real Patronato sobre Discapacidad.

Gobierno Foral de Navarra (2006). «Ley Foral 15/2006, de 14 de diciembre, de Servicios Sociales». Boletín Oficial de Navarra, 152 (20 dic 2006). Disponible en: <http://www.lexnavarra.navarra.es/detalle.asp?r $=10855>$

—(2010). Departamento de Salud. «Registro de casos de esclerosis múltiple». Pamplona: Gobierno de Navarra.

Huete, A. y Díaz, E. (2009). Estudio sobre situación de necesidades sociosanitarias de las personas con enfermedades raras en España. Madrid: Federación Española de Enfermedades Raras.

Instituto de Investigación de Enfermedades Raras (2011). [en línea]. Madrid: Instituto de Salud Carlos III [con acceso 05-11-2011]. Disponible en: <http $: / /$ isciii.es $>$. En la actualidad remite al sitio <http ///www.orpha. net $>$ para lo relacionado con la información detallada sobre enfermedades raras.

Instituto Nacional de Estadística (2008) [en línea]. «Encuesta sobre Discapacidades, Autonomía personal y situaciones de Dependencia: EDAD 2008». Madrid: INE; [acceso 10-01-2013]. Disponible en <http://www.ine. es $/$ jaxi/menu.do?L=0\&type $=$ pcaxis $\&$ path $=/ \mathrm{t} 15 / \mathrm{p} 418 \&$ file $=$ inebase $>$

- (2010) [en línea]. Encuesta de Condiciones de Vida 2010. Madrid: INE; [acceso 10-01-2013]. Disponible en $<$ http://www.ine.es $/$ jaxi/menu.do?type $=p$ caxis $\&$ file $=$ pcaxis $\&$ path $=\% 2 \mathrm{Ft} 25 \% 2 \mathrm{Fp} 453 \% 2 \mathrm{~F} \% 2 \mathrm{Fa} 2010>$

- (2011) [en línea]. «Encuesta de Presupuestos Familiares 2011». Madrid: INE; [acceso 10-01-2013]. Disponible en: <http://www.ine.es/jaxi/menu. do?type $=$ pcaxis $\&$ path $=\% 2 \mathrm{Ft} 25 / \mathrm{p} 458 \&$ file $=$ inebase $\& \mathrm{~L}=0>$

Kurtzke, J.F. (1983). «Rating neurologic impairment in multiple sclerosis: an expanded disability status scale (EDSS)». Neurology (Cleveland), 33: 14441452 .

- (1993) «Epidemiologic evidence for multiple sclerosis as an infection». Clin Microbiol Rev., October; 6 (4): 382-427.

Kurtzke, JF. (1997). «The epidemiology of multiple sclerosis». En: RaIne, C.S.; McFarland, H.F; Tourtellotte, W.W. (eds). Multiple sclerosis clinical and pathogenetic basis. London: Chapman \& Hall Medical: p. 91-140.

Mallada J. (1999) «Epidemiología de la esclerosis múltiple en España: datos de prevalencia e incidencia». Rev Neurol., 29 (9): 864-7.

Multiple Sclerosis International Federation (2002). Principios para promover la calidad de vida de las personas con esclerosis múltiple. Madrid: MSIF. 
Pérez, L.C. (2006). Discapacidad y asistencia sanitaria. Madrid: CERMI.

Organización Mundial de la Salud (1978). Atención primaria de salud: informe de la Conferencia Internacional sobre Atención Primaria de Salud, Alma-Ata, URSS, 1978. Ginebra: OMS (Serie Salud para todos; 1).

- (2009). Documentos básicos: Constitución de la OMS. 47a ed. Ginebra: OMS.

Pineault, R.; Potvin, L. (2003). «Milton Terris's career».J Public Health Policy, 24: $77-81$.

Ponte, C. (2008). [en línea] «Conceptos fundamentales de la salud a través de su historia reciente». En: Salud y ciudadanía. Teoría y práctica de la innovación. Gijón: Centro del Profesorado y Recursos de Gijón; [acceso 15 nov 2011]. Disponible en: <http://85.152.37.8/recursos/2008/SaludyCiudadania.pdf> Raus, J. (1998). «Multiple sclerosis». En: Delves, P.J.; Roitt, I.M. (eds.). Encyclopedia of Immunology. 2a ed. Londres: Academic Press, p. 1786-9.

Simone I.; Carrara, D.; Tortorella, C.; Ceccarelli, A.; Livrea, P. (2000). «Early onset multiple sclerosis». Neurol Sci., 21: 861-3.

World Health Organization Quality of life assessment (1995). «Position Paper from the World Health Organization». Soc Sci Med., 41 (10): p. 1403-09. 